\title{
Genetic and Environmental Influences on Finger-Sucking and Nail-Biting in Japanese Twin Children
}

\author{
Syuichi Ooki \\ Department of Health Science, Ishikawa Prefectural Nursing University, Kahoku, Ishikawa, Japan
}

\begin{abstract}
The purpose of this study was to clarify the genetic contribution to finger-sucking and nailbiting in childhood using the largest databases available on Japanese twins. The subjects were 1131 pairs of 12-year-old twin children, consisting of 1057 males and 1205 females. All data were gathered by maternal questionnaire, and responses to the questionnaire were checked in the medical interview. The prevalence of finger-sucking between 0 to 2 years was $40 \%$ in males and $43 \%$ in females $(p=.0053)$. The prevalence of nail-biting up until the age of 12 years was $28 \%$ in males and $26 \%$ in females (nonsignificant). Concordance rates and polychoric correlations were all higher in monozygotic pairs than in dizygotic pairs, irrespective of the sex combination. Univariate and bivariate genetic analyses using structural equation modeling was performed. The results showed that the proportion of total phenotypic variance attributable to genetic influences was $66 \%$ in males and $50 \%$ in females for finger-sucking, and $50 \%$ in both males and females for nail-biting. A co-occurrence of finger-sucking and nail-biting was observed in $17.7 \%$ of males (tetrachoric correlation: $r=.40$ ) and $15.7 \%$ of females $(r=.32)$, which was attributed partly to common genetic or environmental factors. The proportion of total shared variance explained by genetic factors was $67 \%$.
\end{abstract}

Finger-sucking, mainly thumb-sucking, and nail-biting are both oral habits that occur often in childhood. Finger-sucking occurs in the early months and peaks at about 18 months to 2 years. Most children stop finger-sucking by age 4 , although a few continue to suck their fingers into adulthood (Lubitz, 1992). Nailbiting is also a common oral habit in children and young adults, but is a rare habit in children under the age of 3 years. From the ages of 3 to 6 years, there is a significant increase in the number of children who bite their nails (Leung \& Robson, 1990).

As with many complex behavioral traits or disorders, most finger-sucking and nail-biting cases in the population are probably mild in severity. Among young children, partial or complete recovery from finger-sucking or nail-biting is common. Parents, however, are frequently concerned about their children's repetitive or stereotyped behavior patterns. These habits are important; they are common in children with a normal psychological status, but may also occur in children with developmental problems or emotional difficulties, or may be secondary to physical disorders (Lubitz, 1992).

Despite nearly a century of research activity, the etiology of finger-sucking and nail-biting remains uncertain. The etiologies have been explained mainly in the light of developmental psychology. Often, finger-sucking is simply a pleasurable activity unrelated to any underlying emotional or other disturbance (Peterson \& Schneider, 1991). Fingersucking before the ages of 4 to 6 years is typically not considered problematic, although there are dental concerns (Lubitz, 1992; Watson et al., 2002). After this age, however, chronic finger-sucking may lead to dental problems, such as malocclusion and other difficulties, and problems with peers or parents (Friman et al., 1993). Unlike with finger-sucking, the role that anxiety, nervousness, tension or stress play in the etiology of nail-biting has been well documented (Bošnjak et al., 2002; Leung \& Robson, 1990). Moreover, imitation of other family members, transference from the finger-sucking habit, and poorly manicured nails in the etiology of nail-biting have also been pointed out (Leung \& Robson, 1990). Contrary to other habitual behaviors in childhood, genetic studies of finger-sucking and nail-biting have seldom been performed.

The aim of this study was to describe the prevalence of finger-sucking and nail-biting in normally developing twin children in Japan, and to clarify the genetic contributions of each trait and the relationship between the two traits.

\section{Received 11 January, 2005; accepted 11 April, 2005.}

Address for correspondence: Syuichi Ooki, Department of Health Science, Ishikawa Prefectural Nursing University, Tsu7-1 Nakanuma, Kahoku, Ishikawa, 929-1212, Japan.E-mail: sooki@kj8.so-net.ne.jp 


\section{Materials and Methods Outline of the Present Samples}

The present sample consisted of volunteer-based Japanese twin databases. These databases were constructed for the purpose of providing maternal and child care assistance to families with multiples, and as databases for genetic epidemiologic twin family studies. The basic characteristics of the databases have been reported in detail elsewhere (Ooki \& Asaka, 2005). The subjects were a total of 1131 twin pairs, consisting of 1057 males and 1205 females in the sixth grade of primary school, all of whom were applicants to the secondary education school attached to the faculty of education at the University of Tokyo from 1981 through 2004 (birth year ranged from 1968 to 1992). At the time the data were collected, most of the twins were 12 years old.

\section{Zygosity Classification}

The zygosity of the twins was determined primarily by questionnaire (Ooki \& Asaka, 2004), which was completed by the mothers. The zygosity types were monozygotic (MZ), unclassified (UZ) and dizygotic (DZ), according to a similarity score that was calculated using five questions regarding physical similarity and confusion of identity between the twins.

The zygosity of 224 pairs who were actually admitted to the school was determined by many DNA/genetic markers as part of entrance procedure to the school, prior to being determined by questionnaire. Of these 224 pairs, the zygosity of 204 pairs was also determined by questionnaire, though 20 pairs were not classified by questionnaire. When zygosity determination by DNA/genetic markers was regarded as the gold standard, the accuracy of the zygosity questionnaire was 97.5\% (199/204; Ooki \& Asaka, 2004). The accuracy was a trade-off according to the percentage of unclassified pairs.

The pairs were classified as follows: $754 \mathrm{MZ}$ consisting of 336 male-male and 418 female-female pairs; 273 DZ consisting of 77 male-male, 69 female-female, 70 male-female, and 57 female-male pairs; and $104 \mathrm{UZ}$ consisting of 52 male-male and 52 female-female pairs. All twins were analyzed as to the calculation of prevalence. Only those pairs whose zygosity was classified were used for genetic analyses.

\section{Data Collection}

Data were collected through hand-delivered questionnaires. The questions covered family structure, obstetric findings on the mothers, the twins' physical growth and development, habitual behaviors and any behavioral problems the twins had had. One of the parents of each applicant, usually the mother, participated in a medical interview conducted by two or three interviewers (including the present author from 1988 onwards), in which their responses to the questionnaire were carefully checked.

From these data, the author analyzed fingersucking and nail-biting in childhood. The frequency of finger-sucking between 0 and 2 years and nail-biting up until the age of 12 years was obtained. The mothers identified the frequency from four categories: often, sometimes, rarely or never, or don't know. The occurrence of nail-biting in the parents of these children in their own childhood was also obtained. Since these data were collected as a portion of the data collected to estimate the total growth and development of these twins, more detailed information was not obtained.

One limitation of the questionnaire survey was that strict medical criteria defining finger-sucking and nail-biting were not used. It would have been difficult to obtain correct answers regarding events and behaviors that had, for some subjects, ceased several years prior to the administration of the questionnaire.

\section{Statistical Methods}

The prevalence of finger-sucking and nail-biting was calculated according to sex, including the subjects of unclassified zygosity. Sex difference was tested by the $\chi^{2}$ test.

Parent-offspring transmission of nail-biting was then analyzed. The nail-biting of the parents when they were children was classified according to the number of parents who had this habit, namely 2,1 or 0 . The nailbiting of their offspring (twins) was classified into two categories, often or sometimes or rarely or never. The results were summarized in $3 \times 2$ contingency tables and tested by the $\chi^{2}$ test. This analysis was performed according to the birth order of the twins (first-born or second-born) to avoid the effects of within-pair similarity, which would make the significant relationship between parent and offspring more detectable.

Next, genetic analysis was performed using $M Z$ and DZ pairs with the complete data on the frequency of the two traits in both twins as described in detail elsewhere (Ooki, 2005). Twin similarity for ordinal data can be estimated using a concordance rate. In the calculation of concordance rates, the answers were summarized in the form of $2 \times 2$ contingency tables. The following two patterns of categorization were performed: often or sometimes versus rarely or never (pattern 1), and often versus sometimes or rarely or never (pattern 2). Proband-wise concordance rates were calculated as $2 \times \mathrm{Conc} /(2 \times$ Conc + Disconc $)$ and pairwise concordance rates were calculated as Conc/(Conc + Disconc), assuming complete ascertainment, where Conc denotes the numbers of affected concordant pairs and Disconc denotes the numbers of discordant pairs (McGue, 1992).

When the number of unaffected twin pairs is known, the polygenic multifactorial model, assuming a latent variable called liability to the traits can be used. When a certain threshold of liability is reached, the traits become manifest. The correlation of liability was obtained as a polychoric correlation (Neale \& Cardon, 1992). This correlation of the latent liability was calculated directly from the $3 \times 3$ table cross-classifying the affected status of the first and second twin in each twin pair. The basic statistics, concordance 
Table 1

Prevalence Rates of Finger-Sucking and Nail-Biting According to Sex

\begin{tabular}{|c|c|c|c|c|}
\hline & & Males & Females & $p$ \\
\hline & $N$ & 1057 & 1205 & \\
\hline \multirow[t]{4}{*}{ Finger-sucking at age 0 to 2 years } & Often & $182(18.1 \%)$ & $273(23.6 \%)$ & \\
\hline & Sometimes & $224(22.2 \%)$ & $225(19.4 \%)$ & \\
\hline & Rarely or never & $601(59.7 \%)$ & $660(57.0 \%)$ & .0053 \\
\hline & Missing value & 50 & 47 & \\
\hline \multirow[t]{4}{*}{ Nail-biting until 12 years } & Often & $56(5.5 \%)$ & $63(5.3 \%)$ & \\
\hline & Sometimes & $228(22.3 \%)$ & $247(20.9 \%)$ & \\
\hline & Rarely or never & $737(72.2 \%)$ & $871(73.8 \%)$ & ns \\
\hline & Missing value & 36 & 24 & \\
\hline
\end{tabular}

Note: Sex difference was tested by $\chi^{2}$ test.

rates, and polychoric correlations were computed using PC SAS version 8 (SAS, 1999).

The author used structural equation modeling techniques to estimate the variance components and to compare different genetic models by carrying out standard univariate twin analysis (Neale \& Cardon, 1992). As the data of opposite-sex DZ twin pairs were obtained, homogeneity and heterogeneity of the parameters effects on males and females could be compared directly. The male and female parameters are set to be equal in a homogeneity model. The genetic analyses were conducted on the polychoric correlations.

These models postulated four sources of variance in liability to the traits: (1) additive genetic factor (A); (2) nonadditive genetic factor $(\mathrm{D})$; (3) shared environmental factor (C); or (4) nonshared environmental factor (E). The proportion of the total variance in liability due to additive and nonadditive genetic factors and shared and nonshared environmental factors were termed $\mathrm{a}^{2}$, $\mathrm{d}^{2}, \mathrm{c}^{2}$ and $\mathrm{e}^{2}$, respectively, where $\mathrm{a}, \mathrm{d}, \mathrm{c}$ and e denote each path coefficient from latent variables $(A, D, C, E)$ to observed variables. It is possible to fit models based on the different combinations of these four parameters. The best-fitting model was chosen using the information criteria of Akaike (AIC; 1987), the chi-square value minus twice the degree of freedom. The model with the lowest value of AIC reflects the best balance of goodness-of-fit and parsimony.
Next, phenotypic correlations between fingersucking and nail-biting were calculated as polychoric correlations, and bivariate genetic analyses (Neale \& Cardon, 1992) were performed. A Cholesky decomposition was utilized. Structural equation modeling was performed by the Mx software package (Neale, 2000).

\section{Ethical Issues}

Informed consent concerning the statistical analysis of the data was obtained from each twin and his or her parents, mainly in writing, as part of the application process (Ooki \& Asaka, 2005).

\section{$\overline{\text { Results }}$}

\section{Prevalence of Finger-Sucking and Nail-Biting}

The prevalence and number of subjects in each frequency category for the occurrence of finger-sucking and nail-biting in childhood are given in Table 1.

Finger-sucking was significantly $(p=.0053)$ more common in females than in males. There was no sex difference in nail-biting. Finger-sucking and nail-biting were not significantly related to zygosity and birth order, that is, first-born and second-born (data not shown).

\section{Parent-to-0ffspring Transmission of Nail-Biting in Childhood}

The results are summarized in Table 2. A strong parent-offspring relationship was observed irrespective of birth order $(p<.0001)$.

\section{Table 2}

Parent-Offspring Transmission of Nail-Biting

\begin{tabular}{ccccc}
\hline \multirow{2}{*}{ Number of parent(s) who displayed nail-biting in childhood } & \multicolumn{3}{c}{ Nail-biting in twins } \\
\cline { 2 - 5 } & \multicolumn{2}{c}{ Twin 1 (first-born) } & \multicolumn{2}{c}{ Twin 2 (second-born) } \\
\hline 0 & Yes & No & Yes & No \\
1 & $132(52.6 \%)$ & $444(72.0 \%)$ & $131(53.0 \%)$ & $443(71.5 \%)$ \\
2 & $100(39.8 \%)$ & $158(25.6 \%)$ & $95(38.5 \%)$ & $164(26.5 \%)$ \\
& $19(7.6 \%)$ & $15(2.4 \%)$ & $21(8.5 \%)$ & $13(2.1 \%)$ \\
\hline
\end{tabular}

Note: Parent-offspring transmission was tested by $\chi^{2}$ test. 


\section{Concordance in Twin Pairs}

Table 3 shows the pairwise and proband-wise concordance rates. All pairwise and proband-wise concordance rates of $\mathrm{MZ}$ pairs were higher than those observed in $\mathrm{DZ}$ pairs. The ratios of concordance rates, namely, the concordance rate of $M Z$ pairs divided by that of same-sex DZ pairs, were all higher in pattern 2 than in pattern 1.

Based on the $3 \times 3$ contingency tables, polychoric correlations $(r)$ were computed. These results are also shown in Table 3. The correlations of MZ pairs were higher than those of DZ pairs.

\section{Models of Genetic and Environmental Factors}

The results of the univariate genetic analysis are shown in Table 4.

As to finger-sucking, a heterogeneity ACE model was selected as best-fitting model, whereas a homogeneity ACE model did not fit well. The contribution of nonshared environmental factor was relatively small. As to nail-biting, a homogeneity ACE model showed the best fit, though the heterogeneity model also fit well. These results were in some degree expected based on the polychoric correlations. The path coefficients and variance components with $95 \%$ confidence intervals are also shown in Table 4 . In the best-fitting models, the estimated proportion of total phenotypic variance attributed to the genetic compo- nent was $66 \%$ in males and $50 \%$ in females for fingersucking (heterogeneity model), and $50 \%$ in both males and females for nail-biting (homogeneity model). Common environmental factors were also observed regarding both finger-sucking $(26 \%$ as to males and $31 \%$ as to females) and nail-biting (23\% as to both males and females).

\section{Bivariate Genetic Analyses of Finger-Sucking and Nail-Biting}

Co-occurrence between finger-sucking and nail-biting was observed in $17.7 \%(173 / 975)$ of males and $15.7 \%$ (179/1137) of females, when including often and sometimes in one category, as shown in Table 5.

Polychoric correlations calculated using the $3 \times 3$ contingency table were $r=.40$ in males and $r=.32$ in females.

Polychoric correlations according to zygosity were pooled over sex to stabilize the results of model fitting. The MZ pairs showed higher correlations than the DZ pairs in both finger-sucking $(r=.86$ vs. $r=.59)$ and nail-biting $(r=.74$ vs. $r=.47)$. Phenotypic correlations of finger-sucking and nail-biting in the same individual were relatively high $(r=.34$ to .43$)$ irrespective of zygosity and birth-order. The cross-correlations between finger-sucking and nailbiting of the MZ pairs were higher than or as high as those of the DZ pairs $(r=.31$ vs. $r=.17$ or $r=.26$ vs. $r=.27)$. The best fitting model was the bivariate

Table 3

Concordance Rates and Polychoric Correlations of Finger-Sucking and Nail-Biting According to Zygosity and Sex Combination

\begin{tabular}{|c|c|c|c|c|c|c|c|c|}
\hline & & \multicolumn{3}{|c|}{$\mathrm{MZ}$} & \multicolumn{4}{|c|}{$\mathrm{DZ}$} \\
\hline & & \multirow[t]{2}{*}{$\mathrm{MM}$} & \multirow[t]{2}{*}{$\mathrm{FF}$} & \multirow[t]{2}{*}{ Total } & \multirow[t]{2}{*}{$\mathrm{MM}$} & \multirow{2}{*}{\multicolumn{2}{|c|}{$\begin{array}{c}\text { Total } \\
\text { (same-sex) }\end{array}$}} & $\mathrm{FM}$ \\
\hline & & & & & & & & \\
\hline & $N$ (pairs) & 311 & 393 & 704 & 68 & 66 & 134 & 112 \\
\hline \multicolumn{9}{|c|}{ Finger-sucking between } \\
\hline \multirow[t]{7}{*}{0 and 2 years } & Pattern 1 & & & & & & & \\
\hline & Proband-wise & $.89(218 / 245)$ & $.81(280 / 347)$ & $.84(498 / 592)$ & $.69(40 / 58)$ & $.72(42 / 58)$ & $.71(82 / 116)$ & $.66(56 / 85)$ \\
\hline & Pair-wise & $.80(109 / 136)$ & $.68(140 / 207)$ & $.73(249 / 343)$ & $.53(20 / 38)$ & $.57(21 / 37)$ & $.55(41 / 75)$ & $.49(28 / 57)$ \\
\hline & Pattern 2 & & & & & & & \\
\hline & Proband-wise & $.77(92 / 120)$ & $.72(150 / 208)$ & $.74(242 / 328)$ & $.54(14 / 26)$ & $.46(12 / 26)$ & $.50(26 / 52)$ & $.45(14 / 31)$ \\
\hline & Pair-wise & $.62(46 / 74)$ & $.56(75 / 133)$ & $.58(121 / 207)$ & $.37(7 / 19)$ & $.30(6 / 20)$ & $.33(13 / 39)$ & $.29(7 / 24)$ \\
\hline & Polychoric $r$ & .92 & .81 & .86 & .61 & .57 & .59 & .55 \\
\hline
\end{tabular}

Nail-biting until

12 years

\begin{tabular}{llll} 
Pattern 1 & & & \\
Proband-wise & $.72(132 / 184)$ & $.65(144 / 220)$ & $.68(276 / 4)$ \\
Pair-wise & $.56(66 / 118)$ & $.49(72 / 148)$ & $.52(138 / 2$ \\
Pattern 2 & & & \\
Proband-wise & $.55(18 / 33)$ & $.50(22 / 44)$ & $.52(40 / 77)$ \\
Pair-wise & $.38(9 / 24)$ & $.33(11 / 33)$ & $.35(20 / 57)$ \\
Polychoric $r$ & .76 & .72 & .74 \\
\hline dizygotic; MM = male-male; FF=female-female; FM = female-male. \\
etimes vs. rarely or never. \\
times and rarely or never. \\
were calculated according to $3 \times 3$ contingency tables.
\end{tabular}


Cholesky decomposition ACE model (AIC $=-2.941, p$ $=.93$ ). The path coefficients of the best-fitting model are shown in Figure 1. The proportion of total shared variance explained by genetic factors was $67 \%$, which was calculated as $(.73 \times .33) /((.73 \times .33)+$ $(.57 \times .10)+(.37 \times .17))$.

\section{Discussion}

The prevalence rates of finger-sucking and nail-biting reported in the literature have been widely divergent. The reason for this variation partly reflects differences across studies in sample characteristics, for example, subject age, sampling methods (interview vs. questionnaire, self-reports vs. reports by others), and subject selection (clinic-based vs. population-based), as well as diagnostic criteria (Farsi, 2003; Ravin, 1974). Moreover, the influence of cultural variations on finger-sucking (Lubitz, 1992), including the widespread use of a dummy or pacifier (Ravin, 1974), have been pointed out.

Regarding the prevalence of finger-sucking, between $30 \%$ and $40 \%$ of preschool children (Watson et al., 2002) or $50 \%$ of children in total (Lubitz, 1992) develop this behavior. According to Ravin (1974), the prevalence of a sucking habit, including finger-sucking, varied between $61 \%$ and $87 \%$ in nine reports on the subject. Regarding the prevalence of nail-biting, Leung and Robson (1990) stated in a review that $28 \%$ to $33 \%$ of children aged 7 to 10 years were nail-biters. Nail-biting was observed in 9\% (96/1025, including other biting) of 6- to 11-year-old children (Bošnjak et al., 2002), in $23 \%(91 / 394)$ of children aged 9 to 14 years (Sari \& Sonmez, 2002), and in 28\% (308/1113) of children aged 3 to 15 years (Farsi, 2003). There have been few reports, however, on the prevalence of these habits in twins. Of them, Bakwin (1971a) reported that 30\% (203/676) of twins aged 6 to 18 years engaged in nailbiting. The prevalence of finger-sucking and nail-biting in the present sample was within the range of these various reports. With regards to the sex difference in the prevalence of both finger-sucking and nail-biting, the results reported are inconsistent.

The familial transmission of nail-biting was recognized decades ago. According to Bakwin (1971a), the prevalence of nail-biting in offspring decreases according to the number of parents who were nail-biters during their own childhood. It has been suggested that children learn to bite their nails by imitating their parents, but this is not a valid explanation, as parents usually stop the habit before their children are born (Bakwin, 1971a). The prevalence in the children of parents who had been nail-biters was almost three times greater than in children whose parents (both parents) had not been nail-biters (Bakwin, 1971a). The present results also provided good support for these tendencies. If both parents were nail-biters in childhood, their children showed about a three to four times higher prevalence of nail-biting than children 
Table 5

Co-occurrence Between Finger-Sucking and Nail-Biting

\begin{tabular}{|c|c|c|c|c|c|c|}
\hline \multirow{3}{*}{$\begin{array}{l}\text { Finger-sucking between } \\
0 \text { and } 2 \text { years }\end{array}$} & \multicolumn{6}{|c|}{ Nail-biting until 12 years } \\
\hline & \multicolumn{3}{|c|}{ Males } & \multicolumn{3}{|c|}{ Females } \\
\hline & Often & Sometimes & Rarely or never & Often & Sometimes & Rarely or never \\
\hline Often & 15 & 65 & 99 & 31 & 71 & 164 \\
\hline Sometimes & 21 & 72 & 122 & 11 & 66 & 143 \\
\hline Rarely or never & 14 & 81 & 486 & 18 & 105 & 528 \\
\hline \multicolumn{4}{|c|}{ polychoric $r=.40$} & & & polychoric $r=.32$ \\
\hline
\end{tabular}

whose parents had not had this habit, as shown in Table 2.

Twin studies can directly show the genetic contribution made by parents. Questions concerning knowledge of zygosity determination based on DNA/blood testing were also asked during the medical interview conducted on the present subjects. It was confirmed that most mothers of the same-sex twin pairs did not have much knowledge of their twins' zygosity. Moreover, zygosity misclassification at birth due to findings made from studying placenta frequently occurs in Japan (Ooki et al., 2004). Therefore, mothers of same-sex twins often did not know the correct zygosity of their children. Taking all these things into consideration, the higher similarity in $\mathrm{MZ}$ pairs compared to DZ pairs strongly suggests a genetic contribution to both finger-sucking and nail-biting.

A twin study of nail-biting using large samples has only been performed by Bakwin (1971a). He analyzed 203 twins of 338 same-sex twin pairs who showed nail-biting, and found higher pairwise concordance rates in $\mathrm{MZ}$ pairs than in same-sex DZ pairs. Moreover, he found a clearer difference in the concordance rates between MZ and same-sex DZ pairs when only severe nail-biters were analyzed. These findings were in good accordance with the present study. The genetic contribution to severe nailbiting may be higher than to the usual type of nail-biting. Bakwin (1971b) also performed a twin study of persistent finger-sucking, defined as fingersucking after 3 years, using the same sample. He found a higher pairwise concordance rate in MZ pairs than in DZ ones, although it was not statistically significant. Including the information from opposite-sex DZ pairs in the present study permitted direct detection of the sex-specific effects by structural equation modeling. The results showed that the proportion of total phenotypic variance attributable to genetic influences was $66 \%$ in males and $50 \%$ in females regarding finger-sucking at 0 to 2 years, and $50 \%$ in both males and females regarding nail-biting until age 12 .

Finger-sucking and nail-biting were apt to cooccur for both males and females. According to

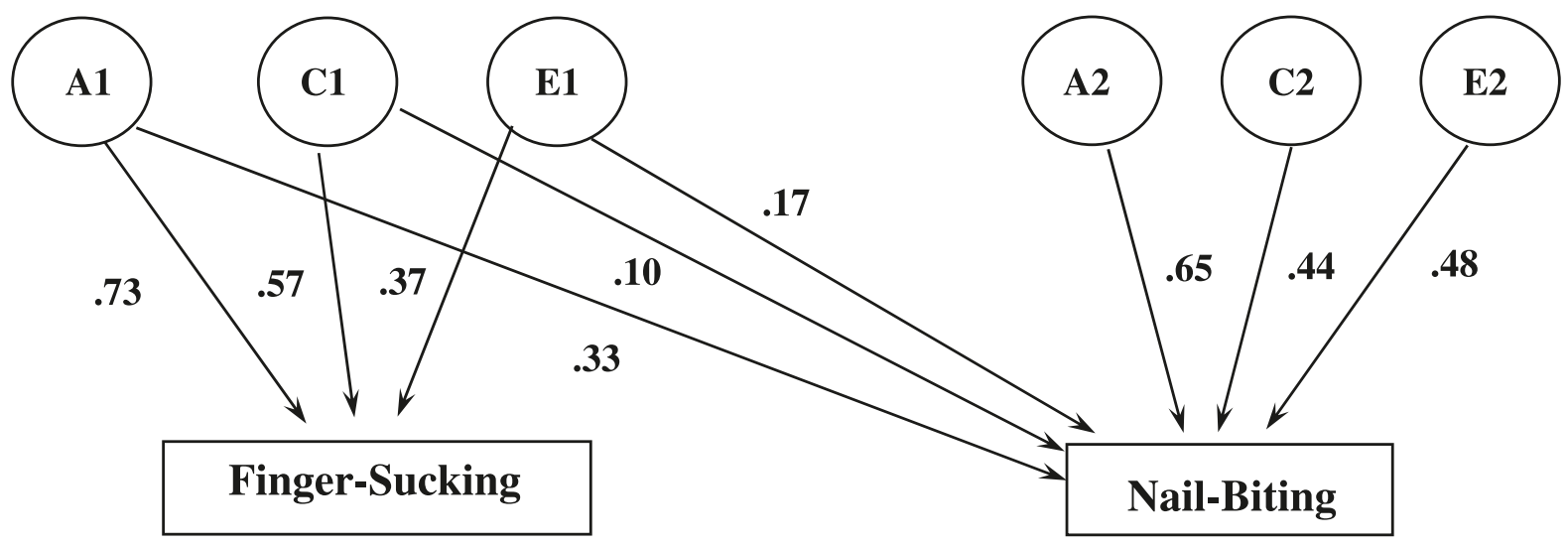

Figure 1

Path coefficient of best-fitting Bivariate Cholesky Decomposition Model.

$A=$ additive genetic factor $;=$ shared environmental factor; $E=$ nonshared environmental factor. The proportions of the total variance in liability due to additive genetic, shared environmental and nonshared environmental factors are calculated by squaring each path coefficient from latent variables $(A, C, E)$ to observed variables. 
Leung and Robson (1990), nail-biting may follow finger-sucking, and the habit serves a need for oral gratification. This chronological relationship is supported by a longitudinal study of children from 2 to 5 years of age (Leung \& Robson, 1990). In Japan, the Ministry of Health, Labor and Welfare has been performing a nationwide survey of child development every 10 years at the change of each decade to compile and present growth and developmental standards for children. On this survey, parents report on behavioral habits which they find annoying (Nihon syoni hoken kyokai, 2002). The results show that in children ages 1 to 6 , finger-sucking decreases with age whereas nail-biting increases with age. This tendency is explained partly by the fact that nail-biting follows finger-sucking. However, a bivariate twin study between finger-sucking and nail-biting has never been conducted. The present results suggest that nail-biting following finger-sucking is partly explained by common genetic and environmental factors for these two traits. The proportion of total shared variance explained by genetic factors was $67 \%$.

The limitations of this study are as follows. First, the prevalence was calculated only by retrospective maternal reports, not by observation, and, therefore, some unexpected bias may be involved. Second, the age effect was not taken into consideration. Since behavioral characteristics in childhood vary greatly with age, it is necessary to increase the accuracy of information about the age of occurrence and duration. The age of the children in the present sample was almost 12 years, and these subjects had, for the most part, already stopped the target behaviors. Third, the total MZ/DZ ratio of the present sample (in which the birth year ranged from 1968 to 1992) was 2.76 (754/273), which was beyond the upper limit of the recent Japanese $\mathrm{MZ/DZ}$ ratio, decreasing from 1.90 (1979) to 1.09 (1994; Imaizumi \& Nonaka, 1997).

In spite of these limitations, the present study indicated significant genetic effects on the occurrence of finger-sucking in early childhood and nail-biting until the age of 12 years. Moreover, it was suggested that the etiology of the co-occurrence of nail-biting following finger-sucking was in part attributed to the common genetic and environmental background for the two traits.

\section{Acknowledgments}

The author gratefully acknowledges the help of Toshimi Ooma with the analysis of the data. The author also gratefully acknowledges the valuable comments of the reviewer. This work was supported in part by a Grant-in-Aid from the Ministry of Education, Culture, Sports, Science and Technology of Japan (grant number 16590507).

\section{References}

Akaike, H. (1987). Factor analysis and AIC. Psychometrika, 52, 317-332.
Bakwin, H. (1971a). Nail-biting in twins. Developmental Medicine and Child Neurology, 13, 304-307.

Bakwin, H. (1971b). Persistent finger-sucking in twins. Developmental Medicine and Child Neurology, 13, 308-309.

Bošnjak, A., Vucicevic-Boras, V., Miletic, I., Bozic, D., \& Vukelja, M. (2002). Incidence of oral habits in children with mixed dentition. Journal of Oral Rehabilitation, 29, 902-905.

Farsi, N. M. (2003). Symptoms and signs of temporomandibular disorders and oral parafunctions among Saudi children. Journal of Oral Rehabilitation, 30, 1200-1208.

Friman, P. C., McPherson, K. M., Warzak, W. J., \& Evans, J. (1993). Influence of thumb sucking on peer social acceptance in first-grade children. Pediatrics, 91, 784-786.

Imaizumi, Y., \& Nonaka, K. (1997). The twinning rates by zygosity in Japan, 1975-1994. Acta Geneticae Medicae et Gemellologiae, 46, 9-22.

Leung, A. K., \& Robson, W. L. (1990). Nailbiting. Clinical Pediatrics, 29, 690-692.

Lubitz, L. (1992). Nail-biting, thumb sucking, and other irritating behaviours in childhood. Australian Family Physician, 21, 1090-1094.

McGue, M. (1992). When assessing twin concordance, use the probandwise not the pairwise rate. Schizophrenic Bulletin, 18, 171-176.

Neale, M. C. (2000). Statistical modeling with Mx. Richmond, VA: Department of Psychiatry, Medical College of Virginia.

Neale, M. C., \& Cardon, L. R. (1992). Methodology for genetic studies of twins and families. Dordrecht, the Netherlands: Kluwer Academic.

Nihon syoni hoken kyokai (Child health care association in Japan). (2002). Child health care based on the new Maternal and Child Health Handbook in 2002 and health research for the children aged 0-6 years by the Ministry of Health and Welfare of Japan in 2000. In T. Kawai \& M. Hirayama M (Eds.), Child health care series No. 55. Tokyo, Japan: Nihon syoni hoken kyokai.

Ooki, S. (2005). Genetic and environmental influences on stuttering and tics in Japanese twin children. Twin Research and Human Genetics, 8, 69-75.

Ooki, S., \& Asaka, A. (2004). Zygosity diagnosis in young twins by questionnaire for twins' mothers and twins' self-reports. Twin Research, 7, 5-12.

Ooki, S., \& Asaka, A. (2005). Comparison of obstetric and birthweight characteristics between the two largest databases of Japanese twins measured in childhood. Twin Research and Human Genetics, 8, 63-68.

Ooki, S., Yokoyama, Y., \& Asaka, A. (2004). Zygosity misclassification of twins at birth in Japan. Twin Research, 7, 228-232. 
Peterson, J. E., \& Schneider, P. E. (1991). Oral habits: A behavioural approach. Pediatric Clinics of North America, 38, 1289-1307.

Ravin, J. J. (1974). The prevalence of dummy and fingersucking habits in Copenhagen children until the age of 3 years. Community Dentistry and Oral Epidemiology, 2, 316-322.

SAS (1999). SAS/STAT user's guide (Version 8). Cary, NC: SAS Institute, Inc.
Sari, S., \& Sonmez, H. (2002). Investigation of the relationship between oral parafunctions and temporomandibular joint dysfunction in Turkish children with mixed and permanent dentition. Journal of Oral Rehabilitation, 29, 108-112.

Watson, T. S., Meeks, C., Dufrene, B., \& Lindsay, C. (2002). Sibling thumb sucking. Effects of treatment for targeted and untargeted siblings. Behavior Modification, 26, 412-423. 